This item was submitted to Loughborough's Research Repository by the author.

Items in Figshare are protected by copyright, with all rights reserved, unless otherwise indicated.

\title{
Special relativity: interpretation and implications for space-time geometry
}

PLEASE CITE THE PUBLISHED VERSION

PUBLISHER

Professional Engineering Publishing / @ IMECHE

VERSION

VoR (Version of Record)

LICENCE

CC BY-NC-ND 4.0

REPOSITORY RECORD

Rahnejat, Homer. 2019. "Special Relativity: Interpretation and Implications for Space-time Geometry". figshare. https://hdl.handle.net/2134/4802. 
This item was submitted to Loughborough's Institutional Repository (https://dspace.lboro.ac.uk/) by the author and is made available under the following Creative Commons Licence conditions.

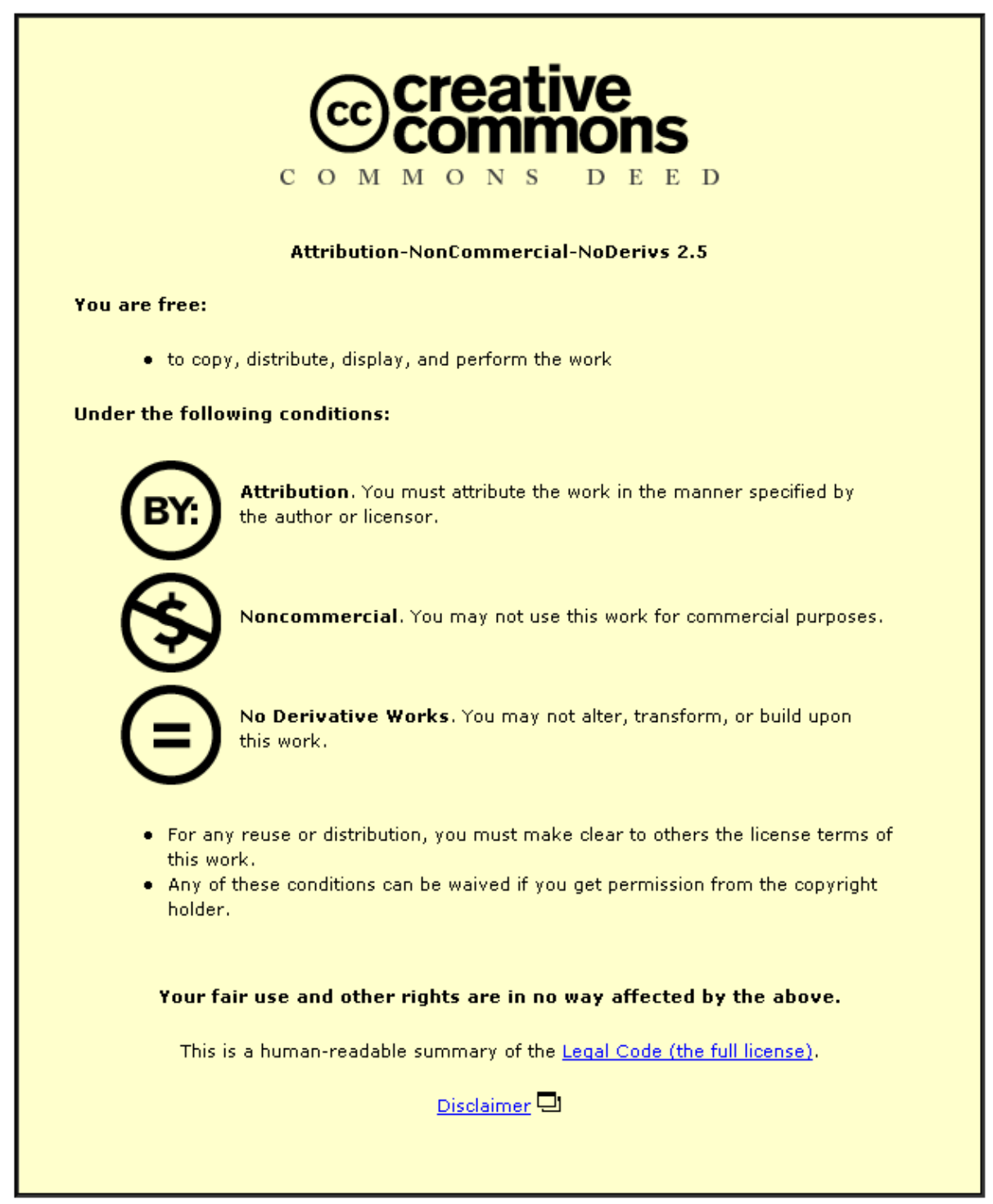

For the full text of this licence, please go to: http://creativecommons.org/licenses/by-nc-nd/2.5/ 


\title{
Special relativity: interpretation and implications for space-time geometry
}

\author{
H Rahnejat \\ Wolfson School of Mechanical and Manufacturing Engineering, Loughborough University, Loughborough, \\ Leicestershire LE11 3TU, UK
}

The manuscript was received on 20 January 2005 and was accepted after revision for publication on 6 April 2005.

DOI: $10.1243 / 146441905 X 10122$

\begin{abstract}
The paper commemorates the centenary of the special theory of relativity, which effectively sets the limit for the structure of space-time to that of the stationary system. The long lasting debate for definition of concepts of instantaneity and simultaneity was thus resolved by the declaration of constancy of speed of light in vacuo as a law of physics. All motions were thus bounded by the light cone and described by the properties of differential geometry, firmly anchored in the calculus of variations. The key contribution underpinning the theory was the resolution of the contradiction imposed by the Galilean transformation through physical explanation and the adoption of the Lorentzian transformation. This highlighted the relative nature of both space and time and the linkage of these to preserve the sanctity of the light cone. The resulting space-time geometry was then founded on the traditional calculus of variation with the addition of this transformation. This retains the time as an independent coordinate and its linkage to space in an explicit form. One implication of this approach has been the retention of the concept of infinitum for some physical quantities as a drawback for use of the Lorentzian transformation. The paper shows that this singular behaviour need not arise if the explicit linkage in space-time is abandoned in favour of the implicit inclusion of time as a link between the curved structure of space and the speed of light, thus restating the calculus of variation in line with special relativity. This points to a closed loop space-matter field, which may belie the fabric of the continuum. One implication of this interpretation is that a small variation in speed of light within the field would be required to dispense with the aforementioned singular nature of the Lorentzian boost, while still remaining within the spirit of special relativity.
\end{abstract}

Keywords: special theory of relativity, principle of incompleteness, closed loop space-matter field

\section{INTRODUCTION}

With the acceptance of the Copernican heliocentric system, it became apparent to Galileo [1] that all bodies were subject to motion, thus measurement of kinematic quantities cannot be achieved in an absolute sense, but relative to an observer, also in some state of motion. He declared this to be the principle of relativity. With this realization, the concept of relativism was born. Galileo then stated that no observer could distinguish between the states of absolute rest and absolute motion.
Measurement of motion of an entity required a transformation to the frame of reference attached to the observer. This transformation was provided by Galileo [1] for observers in relative uniform motion, known as the Galilean transformation in shear, because the world-lines of motion of entities appear as straight lines. At the turn of the 20th century, Galilean transformation was used in an experiment to measure the velocity of the Earth relative to aether (the so-called aether wind), which was assumed to occupy the empty space (see any standard physics text on the Mickelson-Morley 
experiment and section 3). Furthermore, it was noted that Maxwell's equations for electromagnetic waves do not transform properly under Galilean transformation. Lorentz [2] had already established that at velocities nearing the speed of light, the measured distances in the direction of motion appear to significantly contract (referred to as the Fitzgerald's Contraction, who first described this phenomenon). Lorentz provided a new form of transformation, which took this anomaly into account and enabled proper transformation of Maxwell's equation [3]. Lorentzian transformation, referred to as the boost function, provides a relationship for time of an observer as a mixture of time and spatial coordinates of another. Thus, not knowing the physical implications of this relationship, he rather uncomfortably called the time of an observer the local time. An important feature of the Lorentzian transformation is that it limits motion of observable matter within the light-cone. While much of the physics' world at the turn of the 20th century was concerned with the seeming incompatibility of Maxwell's equations with the classical Newtonian physics, with a stroke of ingenuity, Einstein unified kinematics by declaring the constancy of velocity of light in vacuo as a law of physics, and brushed aside the concepts of instantaneity and simultaneity by replacing them with the time of the stationary system (the Galilean absolute rest or absolute motion, see the Editorial to this issue), and declared the Galilean principle of relativity also as a law of physics. Later, he named the theory underpinning these declared laws of physics as the special theory of relativity, recognizing its limited validity to uniform motion of matter [4].

\section{ASPECTS OF CALCULUS OF VARIATIONS}

The foundation of physics of motion, as shown variously here, is based upon differential geometry or simply properties of curves. Additionally, the motion of matter on such curves can be described with regard to infinitesimal changes or evanescent quantities. In other words, small increments of time only are admissible. The study of vanishing changes within differential and integral geometry is known as the calculus of variation. Newton placed the basis of his theories of motion on this approach, using a series of lemmas and corollaries in the style of the early Greek mathematicians such as Euclid and Pythagoras. This approach avoided the rather tedious and rigorous method later suggested by Cauchy, but is equally effective and far more accessible.

Perhaps, the most important and underlying properties of limits in differential geometry are the curvature properties of a path, giving physical meaning to kinematic quantities: velocity and acceleration. For this purpose, Newton introduced Lemmas VI-VII in the Principia [5], using a geometrical proof as depicted in Fig. 1. Note that the angle of contact made at point A should vanish for the curve to continue beyond it. Newton put it as a Lemma in the following form (refer to Fig. 1): 'If any arc ACB, given in position, is subtended by its chord $\mathrm{AB}$, and in any point $\mathrm{A}$, in the middle of continued curvature, is touched by a right line $\mathrm{AD}$, produced both ways; then if the points $\mathrm{A}$ and $\mathrm{B}$ approach one another and meet, I say, the angle $\mathrm{BAD}$, contained between the chord and the tangent, will be diminished in infinitum, and ultimately will vanish.'

The proof provided by Newton makes use of refuting the contrary: that the continuity of the curvature is contradicted if the lemma is not upheld. The extension of this lemma was made by a subsequent one, where Newton stated: 'The same things being supposed, I say that the ultimate ratio of the arc, the chord, and tangent, any one to any other, is the ratio of equality [5].' Then, using elementary

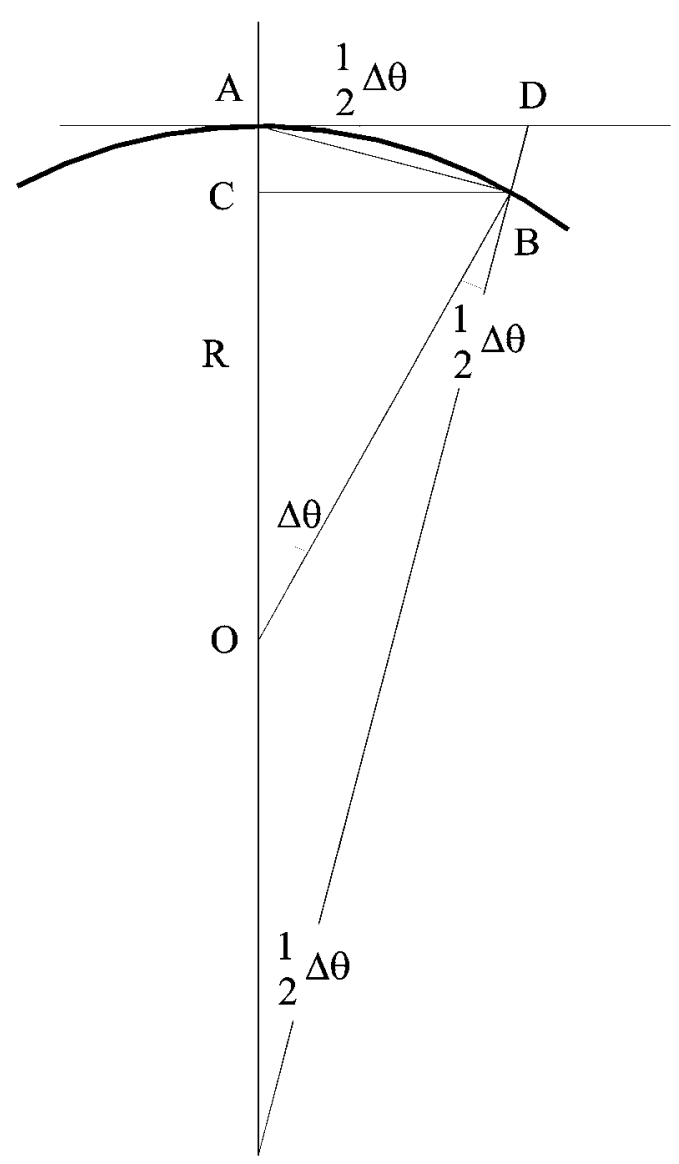

Fig. 1 Limits in the Euclidean geometry 
Euclidean geometry, one can see that (see Fig. 1)

$\operatorname{arc} \mathrm{ACB}=R \Delta \theta$

$$
\begin{aligned}
& \text { chord } \mathrm{AB}=2 R \sin \frac{\Delta \theta}{2} \\
& \text { tangent } \mathrm{AD}=2 R \tan \frac{\Delta \theta}{2}
\end{aligned}
$$

It is clear that as $\Delta \theta \rightarrow 0, \sin \Delta \theta \approx \tan \Delta \theta \approx \Delta \theta$ and the ratio of all these evanescent quantities tend to unity. If one is to assume that point $\mathrm{B}$ converges upon $\mathrm{A}$ in a uniform manner with a velocity, $v$, and a correspondingly short time interval $\Delta t \rightarrow 0$, then $R \Delta \theta=v \Delta t$. This can be represented as $v=R \Delta \theta / \Delta t=\Delta x / \Delta t$, where in Fig. $1, \mathrm{AD}=x$. Thus, in limit with the diminishing quantities

$$
v=\lim _{\Delta t \rightarrow 0}\left(\frac{\Delta x}{\Delta t}\right)
$$

In differential geometry, one can represent this in the limit as $v=\mathrm{d} x / \mathrm{d} t$.

\section{SOME LIMITATIONS WITH DEFINITIONS}

The problem with the earlier mentioned definition of velocity is that $\Delta t \rightarrow 0$, which means that in the extreme case of it attaining the value of zero, the magnitude of velocity reaches infinity. This breaches the velocity limitation set at the speed of the stationary system, in other words, the speed of light. It would be asked naturally, what is wrong with that? The simplest explanation would be: because physics is the study of nature as perceived by humans, and only verifiable as such, and that such observations are bounded by the speed of light, then concepts such as infinite velocity are meaningless. If this view is adopted, then some unnatural conclusions can be drawn from some of the most fundamental axioms in physics. To demonstrate this, the following example is used.

Newton's three axioms form the cornerstone of physics. Using his second axiom, and the same calculus of variation as mentioned earlier

$$
F=m a=m \frac{\mathrm{d} v}{\mathrm{~d} t}
$$

The velocity is

$$
\int \mathrm{d} v=\frac{F}{m} \int \mathrm{d} t
$$

or

$$
v=\frac{F}{m} t+C
$$

and choosing the boundary conditions $v=\left.0\right|_{\mathrm{t}=0}$, $\mathrm{C}=0$, thus $v=(F / m) t$.

For any small ratio of $F / m$ (corresponding to an inappreciable force applied to a very massive body), as long as sufficient time is allowed, $t \rightarrow \infty$, $v \rightarrow \infty$, which is simply not sensible.

This simple example, among others, meant that Einstein's special theory of relativity should be upheld. The special theory of relativity simply states that velocity is limited by the speed of propagation of light in vacuo [4]. It further confirms the Galilean principle of relativity, which draws no distinction between the state of absolute rest and absolute motion, both of which are representations of the speed of light in vacuo.

To correct the previously mentioned anomalous problem and at the same time retain the Newtonian axioms, it should be noted that the mass of the test entity is related to its velocity. Therefore, the special theory of relativity implies that as the velocity of the entity progressively increases, so should its resistance to motion. This is also a restatement of the Galilean principle of inertia [1]. Thus, mass of an entity is not a constant as treated earlier, but a function of the state of its motion (say velocity). One can state this simply as $m=f(v)$. An important conclusion is that mass is a motion-like property, and it should be possible to describe it in terms of differential geometry, like the kinematic quantities. How does this affect Newton's second axiom? Because

$$
F=m a=\frac{\mathrm{d}(m v)}{\mathrm{d} t}=\frac{\mathrm{d}}{\mathrm{d} t}(f(v) v)
$$

which means that

$$
F \int \mathrm{d} t=\int \mathrm{d}(f(\nu) v)
$$

This is simply the impulse-momentum equation in which for a given impulse, the momentum should be conserved. This means that an observer would note an increasing resistance to the motion of the entity, if its velocity were to progressively increase, as suggested earlier. This means that its mass also appears to increase, although such will not be noted by the entity itself. Thus, mass, like other motion-like quantities, has a relativistic nature. The key point is that it is not merely the simple traditional interpretation put upon its definition, and as yet it is not fundamentally understood. Nevertheless, to carry out the integration in the earlier equation, one would need to know the function $f(v)$ apriori.

Without going into a detailed historical review, it can be noted that the way an observer perceives the velocity of a moving object, $v$, is relative to the speed of observation itself (i.e. the speed of light, $c$ ). 
Thus, if a beam of light is directed at an object moving away from an observer, the velocity of the beam relative to it will be $(c-v)$. When the beam reflects from this object and returns to the observer, then it would be $(c+v)$. It is clear that from the average speed, the results obtained would be $c$. Thus, any variations in the velocity of the object, $v$, would be insensible. The problem is in the way the transformation is made from the frame of the moving object to that of the observation itself. This transformation is referred to as the Galilean transformation in shear, and was noted in an attempt to measure the velocity of the Earth relative to the so-called aether in the Michelson-Morley experiment (readers are referred to any standard text book on the physics of motion).

It is, therefore, obvious that with the Galilean transformation, the irrational conclusion would be that $v=0$ always. Subsequent to the MichelsonMorley experiment, it became clear that a new transformation was needed between the space-time coordinates of a moving object $\left.X^{i}\right|_{i=0 \rightarrow 3} \equiv(t, x, y, z)$ and those of an observer $\left.\xi^{j}\right|_{j=0 \rightarrow 3} \equiv(\tau, \eta, \varsigma, \zeta)$. The Lorentzian transformation [2] shows that when the distance moved by the object is, for example $z$, this appears to be foreshortened to $z \sqrt{1-\left(v^{2} / c^{2}\right)}$ relative to the observer [4]. Of course, when $v \ll c$, then the observer will measure the same actual distance. Otherwise, if the object has moved by a distance, $z=v t$, the observer measures the same end point as $\zeta=z \sqrt{1-\left(v^{2} / c^{2}\right)}+v t$. The same relationship can be obtained for all other spatial coordinates, thus

$$
\xi^{j}=\frac{X^{i}-v X^{0}}{\sqrt{1-\left(v^{2} / c^{2}\right)}}, \quad i=j=1 \rightarrow 3, \quad X^{0}=t
$$

The relationship in the temporal sense is given as

$$
\tau=\frac{z-v t}{\sqrt{1-\left(v^{2} / c^{2}\right)}}
$$

Therefore, the Lorentzian transformation embodies the relative nature of both space and time. The transformation function, $B_{v}=1 / \sqrt{1-\left(v^{2} / c^{2}\right)}$, represents the velocity boost as the velocity of an object nears that of the speed of light [2]. Using this transformation, it is obvious that like space and time, mass is also a relative quantity, and it transforms in the same manner as them (using the principle of preservation of four- momenta: one in time and three in space, see standard texts), thus

$$
m=f(\nu)=\frac{\mu}{\sqrt{1-\left(v^{2} / c^{2}\right)}}
$$

where $\mu$ is the rest mass of the entity. Mass of a moving object is perceived to increase dramatically by an observer as it moves close to the speed of light. This relationship suggests a relativistic mass function of the form shown in Fig. 2.

This so-called definition for mass has certain appeal, in that it affords it a motion-like property, in line with properties of differential geometry. However, it seemingly provides it with no fundamental physical meaning (i.e. the underlying reason for it). Using the definition in equation (10), it is clear that the energy of matter is given as [6]

$$
E=m c^{2}=\frac{\mu c^{2}}{\sqrt{1-\left(v^{2} / c^{2}\right)}}
$$

which leads to the interchangeability of energy and mass, where clearly the quantity $\mu c^{2}$ is the rest energy of matter, implying an intrinsic property, for which no physical explanation can be made. The concept is not appealing to all because it removes from mass, the motion-like property that it afforded by the virtue of equation (10). It also demands the acceptance of point-like (atomistic) nature of mass at the limit $v=0$ or rest $\Delta t=0$, which is beyond the attainable increments of successive observations or $\Delta t \rightarrow 1 / c$. This last point makes the concept of rest mass as an intrinsic quantity philosophically imponderable, something that has concerned both schools of thought, positivist atomism (as in the Cartesian School, see D'Alembert [7]) and rational empiricism of the field theorists (relativism) [8]. The problem is that although relativity affords mass a motionlike property, it does not tie it down explicitly to space, time, or space-time geometry. This would be the main reason for representatives of both the D'Alembert and Mach schools not accepting the theory of relativity in its entirety, observing its hybrid nature with respect to the retention of mass as an intrinsic property. To retain most of the

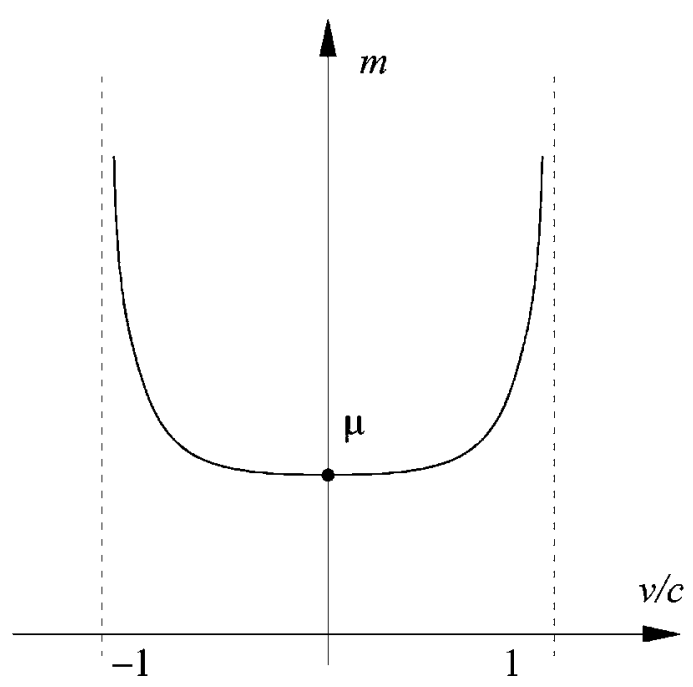

Fig. 2 Relativistic mass function 
foregone discussion within the theory of relativity, there must be a linking between the matter and space-time in the form of a closed field. The calculus of variation needs to be revisited to set the limits in line with the observable (i.e. banishment of concepts such as $v \rightarrow \infty$ and $\Delta t \rightarrow 0$ ).

\section{USE OF LIGHT CONE AND HYPERBOLIC GEOMETRY}

There is a certain elegance in the axiomatic nature and mathematical simplicity of Euclidean geometry. As a result, when finally a link was established between the kinematic quantities and thus force, momentum, and energy with geometry, it furnished them with the same attributes. The axiomatic nature of Euclidean geometry is due to its affinity with human observation, in other words, the Euclidean eyes. Whether this attribute is intrinsic to human nature or one evolved through power of suggestion is beyond the scope of this discourse, but is nevertheless interesting. In any case, it does reveal its shortcomings, when dealing with the problems highlighted earlier (i.e. the perceived increasing mass of an object at very high speeds by an observer, and the problem with the MichelsonMorley experiment). Almost all every day phenomena on the Earth deviate substantially from the speed of light, and this makes the Euclidean geometry akin to human observations. When attempts are made to extend the same to those events (generally of electromagnetic nature) using Euclidean geometry, its limited nature provides rather surprising results, such as those described earlier; the main reason being the breach of the light cone.

Therefore, it became clear that a new form of geometry should be adopted to complement the Lorentzian transformation. Note that the Lorentzian transformation was used to correct the Euclidean observations to fit the facts. This led to the introduction of hyperbolic geometry (a form of nonEuclidean geometry) by Minkowski, with the norm [9], $\|E\|=\left(\xi^{0^{2}}-\Sigma_{i=1 \rightarrow 3} \xi^{i^{2}}\right)^{1 / 2}$, where an event $E$ in $(1+3)$ space-time is defined as

$$
\begin{aligned}
& f(E)=c^{2} \xi^{0^{2}}-\sum_{i=1 \rightarrow 3} \xi^{i^{2}} \\
& E \equiv\left(\xi^{0}=t, \xi^{i}, i=1 \rightarrow 3\right)
\end{aligned}
$$

This not only empowered the Lorentzian transformation to preserve the light cone $c^{2} \xi^{0^{2}}-\Sigma_{i=1 \rightarrow 3} \xi^{i^{2}}=0$, but also defined the kinematics of the space-time as $f(E)=0$ (light-like), $f(E)>0$ (time-like), and $f(E)<0$ (space-like) events.

Thus, the hyperbolic geometry lays down a solid foundation upon which the special theory of relativity resides, and with the embodied Lorentzian transformation the conservation of four momenta and that of matter-energy is achieved.

However, one problem still remains: no explicit alteration is made to differential geometry. Even though hyperbolic geometry confines the time axis (as described earlier) within the light cone, $\Delta t$ is allowed to diminish to zero in the definition of limits in differential geometry. In other words, the interval of time between two successive observations can diminish beyond the value $1 / c$ in the definition of equation (2), and then be corrected later by the constraints introduced by hyperbolic geometry. This is almost the same as using the Euclidean geometry, and then correcting the results of observations to fit the physical facts via the Lorentzian transformation. Furthermore, the relative nature of events is ascertained with respect to given observers in hyperbolic geometry, thus losing the opportunity of using it as a unified framework. Such would not be the case if all events were measured as deviations with respect to a unified frame of observation, or the speed of light, as this remains a constant for all observers in relative motion. Differential geometry can, therefore, be made affine to special relativity, and deviations from it can be described in line with the general theory. This necessitates, first re-defining the calculus of variation and second introducing appropriate space-matter fields.

\section{CALCULUS OF VARIATION MADE AFFINE TO SPECIAL RELATIVITY}

In this way, proper use can be made of the profound nature of hyperbolic geometry. The re-interpretation of differential geometry within the context of special relativity can simply be described as kinematics relative to the speed of light rather than to any particular observer.

Using hyperbolic geometry in $(1+3)$ space-time, $\left(\xi^{i}\right), i=0 \rightarrow 3$. For light-like behaviour [9]

$$
\left(c \xi^{0}\right)^{2}=\sum_{i=1}^{3}\left(\xi^{i}\right)^{2}=\zeta^{2}
$$

Note that $\xi^{0}=t$. Thus, in the usual symbols, $c^{2} t^{2}=\zeta^{2}$. Hence, $t=(1 / \mathrm{c}) \zeta$, which corresponds to the uniform motion of light in vacuo.

To seek a definition for velocity as a slope in differential geometry and bounded by special relativity

$$
v=\frac{\partial s}{\partial t}=\frac{\partial c s}{\partial \zeta}=c \frac{\partial s}{\partial \zeta}+s \frac{\partial c}{\partial \zeta}=c \frac{\partial s}{\partial \zeta}
$$

for $c$ considered to be constant. Note that $\partial s / \partial \zeta=1$ for light-like behaviour. 
This definition is independent of time in an explicit sense, and where $s$ is the arc length of motion. The key point to bear in mind is that time is now embedded in an implicit manner within a scalar field $\nabla c s$, as described later.

This can be defined as

$$
v=\lim _{\Delta \zeta \rightarrow 1 / c}\left(\frac{\Delta s}{\Delta \zeta}\right)
$$

which is clearly a valid definition for velocity as that set by its instantaneous nature (slope in differential geometry) by Cauchy in 1821 [10]. It has, however, a much more profound meaning that has a closer affinity to natural phenomenon by its axiomatic nature through universal observation. In other words, it defines the instantaneous velocity as being in harmony with the frame of observation (i.e. in simultaneity with speed of observation or the speed of light). This underpins the notion that simultaneity is the same as the speed of the stationary system, in other words, the speed of light. This definition, unlike the time-based definition, is underpinned by the special theory of relativity in its inception.

Now the local curvature provides the acceleration as

$$
\begin{aligned}
a & =\frac{\partial}{\partial t}\left\{\frac{\partial s}{\partial t}\right\}=\frac{\partial}{\partial t}\left\{\frac{\partial c s}{\partial \zeta}\right\}=\frac{\partial}{\partial \zeta}\left\{c \frac{\partial c s}{\partial \zeta}\right\} \\
& =\frac{\partial c}{\partial \zeta} \frac{\partial c s}{\partial \zeta}+c \frac{\partial^{2} c s}{\partial \zeta^{2}}=v \frac{\partial c}{\partial \zeta}+c \frac{\partial}{\partial \zeta} \frac{\partial c s}{\partial \zeta} \\
& =v \frac{\partial c}{\partial \zeta}+c \frac{\partial v}{\partial \zeta}
\end{aligned}
$$

which, because the speed of light is assumed to be independent of spatial location, $\zeta$ provides the usual definition for acceleration as $a=$ $c(\partial v / \partial \zeta)=(\partial v / \partial t)$. However, it should be noted that this relationship is only true for light-like behaviour, and thus, the equality $c(\partial v / \partial \zeta)=(\partial v / \partial t)$ is not generally permitted. This point can be exploited advantageously, because for light and other electromagnetic radiation $a=v(\partial c / \partial \zeta)$, the second term on the right-hand side of equation (16) being $c(\partial v / \partial \zeta)=c(\partial / \partial \zeta)(c(\partial s / \partial \zeta))=c^{2}\left(\partial^{2} s / \partial \zeta^{2}\right)=0$, as $v \rightarrow c$, therefore, $(\partial s / \partial \zeta) \rightarrow 1$ (see equation (14)). This is defined as the slope of unity, or the light-like behaviour, where $a=0$. The first term on the right-hand side of equation (16) will also be zero, unless the speed of light was to alter, for example, in the presence of a strong gravitational field.

Away from light-like behaviour, time-like and space-like characteristics are noted by the properties of the Minkowski norm. In general, $f(E)$ in equation (12) can have any real value, with a positive value corresponding to time-like behaviour. Thus, $c^{2} \xi^{0^{2}}-\zeta^{2}=f(E)$. This relationship describes all material points within the light cone in the frame of reference of an inertial observer $\left(\xi^{0}\right.$, $\zeta \equiv \xi^{i}, i=1 \rightarrow 3$ ). Admitting only small quantities, the world-curve of the motion can be stated as $c^{2} \mathrm{~d} \xi^{0^{2}}-\mathrm{d} \zeta^{2}=\mathrm{d} \tau^{2}$, where $\mathrm{d} \tau$ is the proper time of a material point in motion in relativity. The proper four-velocity of this material point is given as $(\partial t / \partial \tau, \partial s / \partial \tau)$, where $\mathrm{d} s$ is the arc length of motion in the Euclidean geometry, corresponding to the proper time, $\mathrm{d} \tau$. The term $\partial t / \partial \tau$ is the local time dilation factor. Then, the spatial proper three-velocity components can be found from

$$
\begin{aligned}
u & =\frac{\partial s}{\partial \tau}=\frac{\partial s}{\partial t} \frac{\partial t}{\partial \tau}=\frac{1}{\sqrt{1-\left(v^{2} / c^{2}\right)}} \frac{\partial s}{\partial t} \\
& =\frac{1}{\sqrt{1-\left(v^{2} / c^{2}\right)}} \frac{\partial c s}{\partial \zeta}
\end{aligned}
$$

Note that $u=\partial s / \partial \tau=\left(1 / \sqrt{1-\left(v^{2} / c^{2}\right)} \quad(\partial c s / \partial \zeta)=\right.$ $(v / c) / \sqrt{1-\left(v^{2} / c^{2}\right)}$, which is in line with both the special case given by equation (14) and the Lorentzian transformation [2]. Two important relations also result, $\quad \partial s / \partial t=\partial s / \partial \tau \mid v \ll c$ and $\partial s / \partial \zeta=(1 / c)$ $(\partial s / \partial t) \mid v \rightarrow c$. Note that the slope of unity is achieved by the second of these relations, while in the general case $\partial s / \partial \zeta=(1 / c) \sqrt{1-\left(v^{2} / c^{2}\right)}(\partial s / \partial \tau)$, which yields the same equality as the earlier one for $v \rightarrow c$, and deviates from unity inversely in proportion to the local time dilation factor as $\partial \tau / \partial t$.

Now, the acceleration of this material point can be obtained by differentiation of equation (17), also employing the definition in equation (16) as

$$
\begin{aligned}
a & =\frac{\partial u}{\partial \tau}=\frac{\partial}{\partial \tau}\left(\frac{\partial s}{\partial \tau}\right)=\frac{\partial}{\partial \tau}\left(\frac{\partial t}{\partial \tau} \frac{\partial s}{\partial t}\right)=\frac{\partial t}{\partial \tau} \frac{\partial}{\partial \tau}\left(\frac{\partial c s}{\partial \zeta}\right) \\
& =\frac{\partial t}{\partial \tau} \frac{\partial}{\partial t}\left(\frac{\partial t}{\partial \tau} \frac{\partial c s}{\partial \zeta}\right)=\frac{\partial t}{\partial \tau} \frac{\partial t}{\partial \tau} \frac{\partial v}{\partial t}=\left(\frac{\partial t}{\partial \tau}\right)^{2} \frac{\partial v}{\partial t} \\
& =\left(\frac{\partial t}{\partial \tau}\right)^{2}\left(v \frac{\partial c}{\partial \zeta}+c \frac{\partial v}{\partial \zeta}\right)
\end{aligned}
$$

\section{KINEMATIC QUANTITIES AS INCOMPLETENESS FROM UNIFIED FRAME OF OBSERVATION}

Definition (16) is a special case of equation (18), where in the latter, the proper time is taken into account. Furthermore, in this case the deviation from the slope of unity constitutes $a \neq 0$. In other words, an accelerated motion yields curved space. Therefore, with the exception of perfect straight line motion of slope of unity (i.e. the light-like behaviour), all other motions are curvilinear, with their 
slopes as deviations from the light-like behaviour in accordance with the quantity $\partial \tau / \partial t$. The corresponding local curvature is obtained by assuming the constancy of the speed of light in equation (18) (i.e. $\partial c / \partial \zeta=0)$ as $\partial^{2} s / \partial \zeta^{2}=\left(a / c^{2}\right)(\partial \tau / \partial t)^{2}$. Therefore, the motion of all material points in any region of space, $\zeta$, within a weak gravitational field may be described with respect to the light-like behaviour by a pair of numbers: its deviation from a slope of unity, and its corresponding local curvature: $\boldsymbol{I} \equiv\left(I^{1}, I^{2}\right) \equiv\left((\partial \tau / \partial t),\left(a / c^{2}\right)(\partial \tau / \partial t)^{2}\right)$, where the vector $\boldsymbol{I}$ is hereinafter defined as the incompleteness of the observed motion, defined previously in geometrical terms in reference $[\mathbf{1 1}]$.

The term $c s$ in equation (14) assumes the meaning of kinematic viscosity in the traditional sense, as it has the units of $\mathrm{m}^{2} / \mathrm{s}$ ( $\mathrm{s}$ being the displacement). Of course, in the current definition here, all quantities are of geometric attributes. The definition, therefore, relates to the extension in a given spatial direction or the stretching of a curve (or matter striving to describe a path). If, as earlier, it can be stretched to its limit of unity, it represents a straight line. In other words, the state of motion of matter to traverse such a path endows to such matter a light-like property.

Now referring to equation (18), note that the curvature is defined as the combination of interactions between the moving matter and the fabric of space and the action of light itself. (i.e. the manner in which light propagates in space). This becomes the definition for acceleration, which sits very naturally with fundamentals of observation itself. The final term on the right-hand side of equation (18) is the usual observation model, indicating curvature of motion as viewed by the speed of observation (i.e. the speed of light). If put in the form $c^{2}(\partial t / \partial \tau)^{2}\left(\partial^{2} s / \partial \zeta^{2}\right)=\left(1 /\left(1-\left(v^{2} / c^{2}\right)\right)\right)\left(\partial^{2} s / \partial \zeta^{2}\right)$ it shows the deviation from light-like behaviour as observed by any observer in uniform motion. However, one can use the light-like slope of unity as a frame of measurement and use the defined incompleteness to note that with the constancy of the speed of light $\boldsymbol{I}^{2}=\left(a / c^{2}\right)(\partial \tau / \partial t)^{2}=\left(a / c^{2}\right)\left(1-\left(v^{2} / c^{2}\right)\right)$, which means the slower the material point, the greater the incompleteness, making it easier to discriminate. This is simply axiomatic. Although the value of $c$ in vacuo and on a geometric slope of unity remains constant, in line with special relativity, there is no reason for it not to adhere to other curvatures to facilitate observation of other phenomena, indicating its wave-like motion.

\section{CLOSED LOOP SPACE-MATTER FIELD}

The earlier mentioned relationships are kinematic in nature. In other words, they are concerned with observation of phenomena by an observer. This means that the inseparability of space and matter is not strictly embodied in them, which accounts for the underlying cause of accelerated motion. By the preceding arguments, a curvature in space is created by the action of matter upon it. Because the unitary nature of matter must be refuted as contrary to evolutionary dynamics, then its multiplicity can be described, in its simplest representation, by the presence of motion of a conglomerate of matter, creating the curvatures of space. Now, using equation (18), $\quad a=(\mathrm{d} t / \mathrm{d} \tau)^{2}\left(\partial^{2} c^{2} s / \partial \xi^{2}\right)$, which for motions $v \ll c \rightarrow \mathrm{d} t / \mathrm{d} \tau \approx 1$. The term $c^{2} s$ can be interpreted as the resistance of space (note that cs has the conventional unit of kinematic viscosity as previously described) to the propagation of matter at the speed of light (i.e. the electromagnetic waves). Therefore, it represents a closed loop space-matter field interpretation. It is a closed loop field, because the presence of all matter in such a space is implicit within the quantity $c^{2} s$, as opposed to an atomistic definition, which is based on the presence of discrete masses such as a gravitational source and target bodies. The quantity $c^{2} s$, in conventional terms, has the unit of $\mathrm{m}^{3} / \mathrm{s}^{2}$. In the Newtonian atomistic view (i.e. the discrete action of bodies in the law of universal gravitation), the product $G M$ provides the basis of interpretation and has the same unit. Einstein's weak gravitational field theory is a half way house, where an open loop field is assumed, as the underlying source for the curved space-time.

In the conventional interpretation of gravitational action

$$
a=\frac{\partial \varphi}{\partial \zeta}=\nabla \varphi
$$

where the simplest form of gravitational potential is a gradient form, inversely proportional to the distance from the source, $M$, as $\varphi=-G M / r$. The negative sign is due to the work done by the gravitational field. As the field varies from position to position in space (i.e. $\zeta \equiv \xi^{i}, i=1 \rightarrow 3$ ), equation (19) can be written in its general form as $a=-\nabla \varphi$. Note that the vector field, $a$, does not depend on any target body of mass, $m$, but only on the source mass, $M$. This precisely demonstrates the open loop nature of the conventional interpretation, explained earlier. Similarly, in the closed loop interpretation

$$
\begin{aligned}
a & =\left(\frac{\mathrm{d} t}{\mathrm{~d} \tau}\right)^{2} \frac{\partial^{2} c^{2} s}{\partial \zeta^{2}}=\left(\frac{\mathrm{d} t}{\mathrm{~d} \tau}\right)^{2} \frac{\partial}{\partial \zeta}\left(\frac{\partial c^{2} s}{\partial \zeta}\right) \\
& =\left(\frac{\mathrm{d} t}{\mathrm{~d} \tau}\right)^{2} \frac{\partial \varphi}{\partial \zeta}=\left(\frac{\mathrm{d} t}{\mathrm{~d} \tau}\right)^{2} \nabla \varphi
\end{aligned}
$$


where $\nabla \varphi=\left(\partial \varphi / \partial \xi^{i}\right), i=1 \rightarrow 3$, and in this case $\varphi=\partial c^{2} s / \partial \zeta=c^{2} \nabla s$ for $c$ remaining constant in accord with special relativity.

\section{UNIVERSAL GRAVITATIONAL CONSTANT AND EINSTEIN'S RIPPLES}

In the strictest terms, the results of the earlier two alternative interpretations cannot be equated, as one is atomistic in nature, while the other relies on a continuous closed loop field. However, in cases where the mass of a source is the dominant factor for the curved space-time as in the case of the solar system (i.e. the Sun accounts for nearly 99 per cent of the mass of the solar system), this equivalence may be assumed. Thus, the gravitational potential may be regarded as equivalent to that of the space-matter field, described earlier

$$
c^{2} \nabla s \equiv \frac{G M}{r}
$$

Now, this can lead to a physical description for the universal gravitational constant, noting that $G=$ $\left(c^{2} / M\right) r \nabla s$, where $\nabla s$ is the slope at the location $\zeta \equiv$ $\xi^{i}, i=1 \rightarrow 3$ at a distance $r$ from the source $M$. This may be surmised as a wave-like or ripple motion, proposed by Einstein as it varies with distance $r$. Now the earlier mentioned relationship can be represented as follows

$$
G=\frac{c^{2} r}{M} \nabla s=\frac{E}{M m} r \nabla s
$$

This relationship provides a physical description for $G$ : as the ripple created by a mass $M$ remotely from any locality (at a distance $r$ ), given by $\nabla s$, where matter of total energy $E$ and mass $m$ can reside. This description ties the curved space with the residing matter and the gravitational action of a nearby massive body. For $G$ to be regarded as a constant (which is in line with all measurements of orbital motions in the solar system), such systems should possess a massive body and $\nabla s$ should be almost insensible, which, as evidence suggests, is the case. Using equation (22), the ripple amplitude caused on the space proximate to the gravitational field of the Earth may be surmised. For example, at a distance of $1000 \mathrm{~km}$ from the surface of the Earth, with $G=6.673 \times 10^{-11} \mathrm{~m}^{3} / \mathrm{kg} \mathrm{s}^{2}$, mass of Earth $=5.976 \times$ $10^{24} \mathrm{~kg}$, its radius $=6371 \mathrm{~km}$ and assuming $c=300000 \mathrm{~km} / \mathrm{s}$, the amplitude of ripple is surmised to be $6 \mathrm{~nm}$. However, it should be noted that the ripple in any spatial location is determined by the action of a many-body system. In the case of a location described earlier, the gravitational potential of the Sun cannot be ignored. If the same relationship was to be used in the case of the Sun's gravitational potential alone, with its mass and distance as $1.989 \times 10^{30} \mathrm{~kg}$ and $1.5 \times 10^{8} \mathrm{~km}$, respectively, then the ripple amplitude obtained is $10 \mathrm{~nm}$. The important point to note is that any object creates ripples in space as it moves and at any location in a system, the ripple amplitude is an amalgam of these constituents, and not necessarily in an additive manner.

To observe why $G$ is regarded as a constant, the relativistic nature of the mass in equation (22) can be replaced, using equation (10). Then

$$
\begin{aligned}
G & =\frac{c^{2}}{M} r \nabla s=\frac{c^{2} \sqrt{1-\left(v^{2} / c^{2}\right)}}{\mu} r \nabla s \\
& \simeq c^{2}\left\{1-\frac{1}{2} \frac{v^{2}}{c^{2}}-\frac{1}{8} \frac{v^{4}}{c^{4}}-\cdots\right\} \frac{r \nabla s}{\mu}
\end{aligned}
$$

It is clear that for massive bodies moving at $v \ll c, G \approx\left(c^{2} r \nabla s / \mu\right)$, which is independent of the state of their motion and that $G$ is of electromagnetic nature (thus a universal constant). It is also a property of the closed field, $c^{2} \nabla s$.

\section{SPECIAL RELATIVITY AND SINGULARITY DUE TO THE LORENTZIAN BOOST}

A problem still remaining, among others, with the approach expounded is retaining the Lorentzian boost function. This can still lead to the attainment of infinitum, for example in the evaluation of $\nabla s$ from equations (22) or (23), where for $v \rightarrow c$, $\nabla s \rightarrow \infty$. The main reason for this is the acceptance of the notion of mass in its traditional atomistic sense. Furthermore, $c^{2} \nabla s$ cannot be claimed as the space-matter field, if mass remains alongside it as a discrete entity. This would indeed be the source of objection by the proponents of both the atomistic school of thought and the field theorists alike. The current exposition favours the latter, while sympathy also exists with D'Alembert's view of the imponderable nature of mass as an entity $[7,8]$.

The most poignant manifestation of the singularity at the heart of the theory of relativity is the Lorentzian boost $\left(1 / \sqrt{1-\left(v^{2} / c^{2}\right)}\right)$, which shows that as $v \rightarrow c, B_{v} \rightarrow \infty$. Thus, many important relations, discussed earlier, including time dilation and relative mass tend to infinity. These are the result of the constraint imposed by the constancy of speed of light in vacuo, or in other words: special relativity. The growing trend in disputing special relativity has come about as a result of a desire to remove this source of singularity, thus extending the theory of relativity, which is felt by many to be the fundamental basis upon which the observed nature resides. Some 
have resorted to observations of accelerated motion of light in vacuo in certain circumstances as anomalous dispersion [12], although Einstein himself had only accepted the same within a gravitational field. If the notion of a closed loop space-matter field, as described above, is accepted then it can be shown that such concept as vacuum has no place since space itself cannot exist in such circumstances. To show this, tidal motion in vacuo must be considered.

In the traditional atomistic view, tidal acceleration created by a source may be defined by derivatives $\partial a / \partial \xi^{i}, i=1 \rightarrow 3$. Using equation (19), this can be represented in terms of the potential as $\partial a / \partial \xi^{i}=$ $\partial^{2} \varphi / \partial \xi^{i} \partial \xi^{j}, i, j=1 \rightarrow 3$, yielding a $3 \times 3$ matrix for $\nabla^{2} \varphi$. At the origin of the source, $\nabla^{2} \varphi=0$ for $i \neq j$. Thus, only the diagonal terms have a finite value. Now, using the Newtonian gravitational potential, it is simple to show that $\nabla^{2} \varphi=0$ (the Laplacian of the potential) at any location in space $\zeta=\xi^{i}, i=1 \rightarrow 3$, away from any source. This is, in fact, the vacuum field equation. In the closed field view of space, the analysis has to be extended to the case of a distribution of matter in order to remain in line with the space-matter field. In this case, $\nabla^{2} \varphi=\nabla \bullet \nabla \varphi=\nabla \bullet a$ (using equation (21)). Note that the product $\nabla \bullet \nabla=$ $\nabla^{2}$ is defined as the inner product of the vector differential operator, thus, $\nabla \bullet a$ is the divergence of $a$. Hence, empty space can be defined as the place, where the average tidal acceleration is given by $\nabla \bullet a=\nabla \cdot \nabla \varphi=c^{2} \nabla \cdot \nabla^{2} \mathbf{s}=c^{2} \nabla^{3} s=0$, when the constancy of speed of light is upheld. Because $c \neq 0$, then $\nabla^{3} s=0$, or the Laplacian $\nabla^{2} s$ is finite. This means that passage of light creates the space, which otherwise will have a null content (see subsequently). For the limiting case of the slope of unity, this is regarded as the theoretical perfection (completeness, $\left(I^{1}, I^{2}\right)=(1,0)$, see section 5 , and also reference $[\mathbf{1 1}])$. In special relativity, this condition is designated the vacuo. In incompleteness, such a space is only created by the passage of light.

This simple analysis qualifies the concept of vacuo, where in this case, it is replaced by the notion of null space: one containing no matter (where the sanctity of inseparability of matter and space is thus upheld). When matter enters a place, space is created, and vice-versa, space must exist as a condition for the presence of matter. Therefore, vacuum in its traditional sense is not the same as the null space. There is a noteworthy subtle difference here. In relativity, the speed of light is constant in vacuo, but in incompleteness, slope of unity remains merely a theoretical perfection in a space where no other matter but light can reside. It may then be surmised that such defined null space is created by the passage of light and ceases to exist without it. To provide some theoretical basis for this, the relations in the preceding paragraph can be investigated again for the general case of the space-matter field, where for $\nabla c \neq 0, \nabla \varphi=v \nabla c+c \nabla v$ (see equations (18) and (20), and note that in general, $\varphi=c v)$. Now

$$
\begin{aligned}
\nabla \bullet a & =\nabla \cdot \nabla \varphi=\nabla \cdot(\nu \nabla c+c \nabla v) \\
& =v \nabla^{2} c+c \nabla^{2} v=0
\end{aligned}
$$

The obvious solution for this empty space, other than for presence of light is $v \rightarrow c$ and $\nabla^{2} v=-\nabla^{2} c$, and noting that $\| \nabla^{2} c||<0$ indicates that $\nabla c$ must attain its maximum value to reach the slope of unity. In incompleteness, a space is created by this action of light, whereas in special relativity, this location represents a form of vacuo.

The boost has now the form

$$
\begin{aligned}
B_{v} & =\frac{1}{\sqrt{1-\left(v^{2} / c^{2}\right)}}=\frac{1}{\sqrt{1-\left(1 / c^{2}\right)\{c(\partial s / \partial \zeta)+s(\partial s / \partial \zeta)\}^{2}}} \\
& =\frac{1}{\sqrt{1-\left(1 / c^{2}\right)\{c \nabla s+s \nabla c\}^{2}}}
\end{aligned}
$$

The first term in the curly brackets is the restatement of the special theory, where in vacuo, and in line with the incompleteness theorem: $\nabla s=1$. If the second term in the curly bracket is ignored, then the singularity in dilation time and the relativistic mass function would be reached. The second term, however, small (and this will always be the case, except for the case of a very strong gravitational field nearby, such as a black hole) is finite, and close examination shows that for proper determinism

$$
\nabla s<1-\frac{s}{c} \nabla c, \nabla c<0, \quad s \neq 0
$$

The first condition states that the slope of unity is merely theoretical, as no other matter than light should reside here. This is also qualified by the arguments in the preceding section, where the maximum value for

$$
\nabla c=0: \nabla s \rightarrow 1 \text { and } \varphi \rightarrow c^{2}(\nu \rightarrow c)
$$

One can surmise that in the absence of matter and any gravitational field, the value of $s \rightarrow \infty$. Astronomical observations do not support this. However, they show large areas of empty space, presumed to be occupied by the so-called grey matter. Speculation should not be favoured, but an equally valid postulate to that of the presence of grey matter can be put forward here, as follows. The Sun's gravitational potential in the vicinity of the Earth is $\sim-8.85 \times$ $10^{8} \mathrm{~m}^{2} / \mathrm{s}^{2}$ and declines within the solar system to $-1.84 \times 10^{7} \mathrm{~m}^{2} / \mathrm{s}^{2}$ in the vicinity of Pluto at its furthest 
orbital position (the negative signs indicate the attractive nature of the gravitational potential). Note that the potential in light propagation in slope of unity, creating space is $\varphi=c^{2}=9 \times 10^{16} \mathrm{~m}^{2} / \mathrm{s}^{2}$, which is much larger than the gravitational potential to bring the matter together, even near a star thousands of times larger than our Sun. Therefore, it may be surmised that wherever the speed of light exceeds that governed by a nearby gravitational field, expansive (or dispersive) action will take place in the space. The creation of this space will push the galaxies apart, as indeed observed by the astronomers. Whether other matter can enter this domain is a question of conjecture. It should be noted that the quantity $c s$, described in section 6 , will assume a very large value, and breaching the required potential to enter this domain appears to be foreboding. Inferring from Einstein's analogy of the fabric of space-time as a warped sheet due to the action of potential fields, a field such as that proposed here is likely to stretch it beyond endurance. Therefore, the alternative proposal to large areas of grey matter may be tears in the fabric of space. As the light nears any significant gravitational field $\nabla c<0$, and its dispersive potential attenuates. This is the reason for the second condition in equation (26), which also further underpins the first stated condition.

Another extreme condition is when $\nabla s \rightarrow 0$ and $\left(s^{2} / c^{2}\right)(\nabla c)^{2} \rightarrow 1$. Thus, $\nabla c / c \rightarrow 1 / s$, which is the limiting case in the first condition in equation (26). The ratio $\nabla c / c$ is the curvature of a ray of light, as shown in the next paragraph (note that it is a negative quantity). Therefore, in this limiting case (using equation (30)) $\nabla \theta \rightarrow 1 / s$. Note from equation (1) that $\nabla \theta=\Delta \theta / \Delta s=1 / R$. Therefore, this limiting condition points to a place, where the curvature of a ray of light adheres completely to the curvature of space, and with $\nabla s \rightarrow 0, s=R \rightarrow 0$ (and both being negative), which is presumably determined by a very strong gravitational field. Now using the general case of the space-matter field potential for this case

$$
\begin{aligned}
\varphi & =c \nu=c(c \nabla s+s \nabla c)=c^{2} \nabla s+c s \nabla c \\
& \rightarrow c s \nabla c=c^{2} s \frac{\nabla c}{c}=c^{2} s\left(-\frac{1}{s}\right)=-\left.c^{2}\right|_{\nabla s \rightarrow 0}
\end{aligned}
$$

It can be observed that the space-matter field potential has an attractive nature under this condition. Equations (27) and (28) specify the bounds of the described potential, $-c^{2}<\varphi<c^{2}$. At the upper limit, uncontrolled dispersion occurs in the converted null space created by the passage of light alone. At the lower bound, the curve of space diminishes. This is interpreted as a singular point with infinite matter. However, at the very limit of potential attaining its lower bound, space cannot exist as shown in equation (28). Thus, electromagnetic radiation from such a location must inhibit this condition to arise. This suggests that blackholes should radiate energy, which conforms to recent observations by Integral, ESA's gamma ray observatory [13], which has shown that black holes at the centre of each galaxy, such as the Sagittarius A-star at the centre of Milky Way act like cosmic vacuum cleaners, drawing in gas and matter trapped due to their large gravitational pull. The matter is then crushed and heated until outward bursts of X-rays and gamma rays take place.

Using equation (28) to represent the boost in terms of the potential $\varphi$, the second term in the radical becomes. $\left(1 / c^{2}\right)\{c \nabla s+s \nabla c\}^{2}=\left(1 / c^{2}\right)\{\varphi / c\}^{2}=\left\{\varphi / c^{2}\right\}^{2}$, and the bounded potential (justified by the earlier discussion) prohibits the attainment of singularity at either of the extreme cases described here. All other phenomena fall in between the two extremes described earlier, for all of which the term in the curly brackets in equation (25) conforms to the conditions in equation (26). Thus, the singularity is removed from the Lorentzian boost function. All that now remains to be addressed is to define mass within the closed loop space-matter field interpretation.

Light propagates in space as a series of wavefronts. Owing to the wave-particle duality interpretation put on the propagating nature of light, its rays are considered as paths described by photons of light, and remain orthogonal to the wavefronts at any given location in the field. By virtue of the principle of incompleteness everywhere in ordinary space the wavefronts are curved, and thus the speed of light must alter from point to point within the field, even though this may be by an inappreciable amount. This argument is in line with the general theory of relativity and also the Huygens' principle [14]. Furthermore, referring to the foregone arguments, it is clear that the speed of light will alter in all such ordinary spaces (spaces other than the defined null space to replace notions of vacuo or empty space). This is true, because the variation of speed of light in gravitational fields and in passage through different media is now well established. Two very proximate locations, $\zeta_{1}$ and $\zeta_{1}+\Delta \zeta$ on a current wavefront can be considered. The new position of this front is considered at the short interval of $\Delta t$, as shown in Fig. 3. The speed of light is considered to be different at these locations according to the Huygens' principle and the corresponding rays of light are shown in the figure. Therefore, the front is slanted (not parallel) with respect to its preceding position. This being the case, there is a small angle between the rays of light from these very proximate locations (i.e. $\Delta \theta$ ). Thus

$$
\Delta \theta \approx \sin \Delta \theta=\frac{c\left(\zeta_{1}\right) \Delta t-c\left(\zeta_{1}+\Delta \zeta\right) \Delta t}{\Delta \zeta}
$$




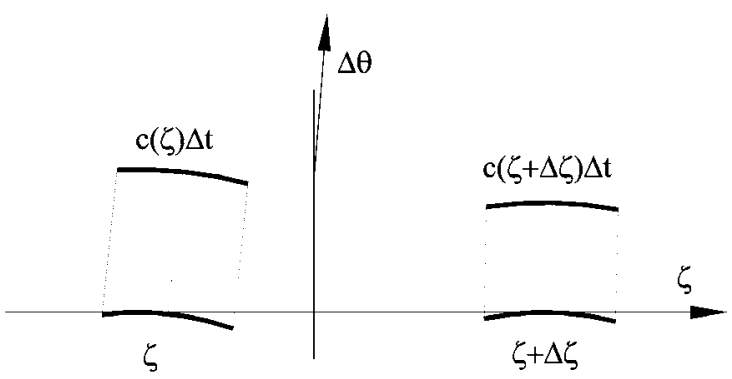

Fig. 3 Bending of light rays

The initial wavefront is taken as a straight line, corresponding to the theoretical limit of $\nabla s \rightarrow 1$, indicating that the rays of light are in transition to an ordinary field. The overall spatial coordinate is $\zeta=$ $\xi^{i}, i=1 \rightarrow 3$ and as shown in section 2 in the limiting case $\Delta t \rightarrow(1 / c) \Delta \zeta$. It follows from equation (27) that in the limit, $c(\mathrm{~d} \theta / \mathrm{d} \zeta)=-\mathrm{d} c / \mathrm{d} \zeta$, or

$$
\nabla \theta=-\frac{\nabla c}{c}
$$

Note that the motion of rays of light in all ordinary spaces (other than the described null space) is decelerative in nature.

\section{INTERPRETATION OF MASS IN THE CLOSED SPACE-MATTER FIELD}

With the singularity removed from the Lorentzian boost function for all matter, the relativistic nature of mass in equation (10) can be retained with the new description of the boost given in the previous section. However, two problems remain. First, a definition is required for the rest mass and second, the atomistic nature of mass should also be abandoned to sit comfortably within the closed loop space-matter field concept.

Because no place in relativistic space-time and within incompleteness is regarded as static, it is clear that the concept of rest mass is merely retained as a mathematical convenience and has no physical meaning or reason. Returning to equation (25), it is clear that for the boost to attain a value of unity

$$
\frac{\nabla s}{s}=\frac{\nabla c}{c}, \quad \nabla c<0
$$

The second condition mentioned earlier has been justified in the previous sections. The first condition states that the curvature of motion at a given location is the same as that of the bending of light rays. Wherever this condition holds, the mass of the object and that regarded as its rest mass are one and the same. Clearly, the curvatures in equation (31) are in the same field, induced by the divergence $\nabla \bullet a$, where by the virtue of Mach's principle the acceleration is due to the presence of all the matter in the field. For the condition given in equation (31), a balance is found between the light propagation field potential and the gravitational field of matter within the field. For this condition

$$
\varphi=c^{2} \nabla s=c^{2} s\left(\frac{\nabla c}{c}\right)
$$

Noting that the Sun accounts for $\sim 99$ per cent of all matter in the solar system, this equality was used in section 8 . Here, it can be noted that equation (23) with the use of the earlier mentioned relationship yields

$$
M=\frac{c^{2} s r}{G}\left\|\frac{\nabla c}{c}\right\|
$$

This relationship provides an atomistic estimate of mass of a closed space-matter field system as determined from a specific location within the field, where the balance described in equation (31) can be assumed. Here, $B_{v}=1, M=\mu$. Because for most fields $B_{v}$ is close to unity, the concept of rest mass assumes the same as the mass itself for them. All parameters in equation (33) are measurable and thus the concentrated equivalent mass of the field can be determined. As regards the field interpretation (or local mass within a given location in the field), it is not necessary to be concerned at all with this, as shown in the following paragraph.

Like D'Alembert, Mach could not accept the atomistic concept of mass as an intrinsic property of matter [15]. In Mach's interpretation, mass of an object is the result of its many interactions with other surrounding matter. This is a relativistic interpretation, except that, for one thing, it was conceived before the theory of relativity and for another, it requires a closed loop field interpretation, which is not a necessary condition in relativity. However, it is precisely in accord with the interpretation put forward here. With Mach's interpretation, as expounded by Einstein

$$
\bar{m}=\frac{m_{1}}{m_{2}}=C \bar{F}=C \frac{F_{1}}{F_{2}}
$$

Now a location in space in such a closed field may be assumed, where a body resides and the condition in equation (31) holds true, and the same body in another location within the same field, where this condition is not upheld, then clearly the ratio: $\bar{m} \neq 1$, and thus using Mach's interpretation $C \propto B_{v}$ in equation (34). It is clear that it is not necessary to be concerned about mass to describe the conditions locally within the field, which is 
completely defined by $B_{v}$ and the field in equation (24), where

$$
\begin{aligned}
& a=\nabla \varphi \\
& \nabla^{2} \varphi=v \nabla^{2} c+c \nabla^{2} v
\end{aligned}
$$

where

$$
v=c \nabla s+s \nabla c
$$

Nevertheless, the earlier arguments suggest that $m \propto 1 / I^{1}$, and for $I^{1} \rightarrow \pm 1, \bar{m}=1$. Thus, one can interpret rest mass as the maximum incompleteness. At the other end of the scale, mass of matter tends to nothing, as indeed is the case for photons of light and nearly all elementary particles such as electrons, where $I^{1} \rightarrow 0$ (i.e. absolute motion). Note that these are measured with respect to the frame of observation (being universal in nature), and not with respect to any particular observer. Mathematically

$$
\begin{aligned}
& I^{1}=\frac{\partial \tau}{\partial t}=\sqrt{1-\left(\frac{\varphi}{c^{2}}\right)^{2}}=\left\{1-\frac{1}{2}\left(\frac{\varphi}{c^{2}}\right)^{2}-\frac{1}{8}\left(\frac{\varphi}{c^{2}}\right)^{4}-\cdots\right\} \\
& \varphi \rightarrow 0 \quad I^{1} \longrightarrow 1 \\
& \varphi \rightarrow \pm c^{2} \quad I^{1} \longrightarrow 0
\end{aligned}
$$

In this interpretation, incompleteness of motion gives rise to a sense of discerning matter by a quantity chosen to be called mass, which is an entirely motion like property, described by the way matter is compelled to move in order to remain on curvatures of space predefined by the presence of all other matter and the local electromagnetic potential of light there. Therefore, elsewhere (other than for conditions in equation (36): $\nabla \varphi \neq 0$. The curvatures of space are defined by the incompleteness, $I^{2}$ (see section 6), where in general

$$
I^{2}=\left(\frac{\nabla c}{c}+\frac{\nabla v}{v}\right)=\frac{a}{c \nu}\left(I^{1}\right)^{2}=\frac{\nabla \varphi}{\varphi}
$$

The ratio $\nabla \varphi / \varphi$ is the curvature of the local potential field. For uniform motion of matter $I^{2}=0$, which relates to absolute rest or absolute motion only. It shows that for the former condition in equation (36) $\|\nabla v / v\|=\|\nabla c / c\|, \nabla c<0$, which means that the curvature of motion of matter at such locations is the same as the curvature of light and electromagnetic radiation. This suggests $\varphi \rightarrow 0$ from equation (36) (an equilibrium between dispersive electromagnetic and local gravitational potentials). These are locations, which demarcate the boundary between electromagnetic radiation and gravitational implosion, such as near the surface of the Sun. This condition is the general case of that described in section 9 , where by virtue of equation (28), $\varphi \rightarrow-c^{2}$, where absolute rest can only theoretically exist.
Thus, for $\varphi>0$, the curvature of the field is positive and all objects are in a propagating field (increasing kinetic energy). Conversely, for $\varphi<0$ there is an increasing stored potential, which is due to gravitation (an increasing mass).

When $I^{2} \neq 0$, non-uniform motions $\left(\nabla_{\varphi} \neq 0\right)$ take place on curvatures created by the presence of a conglomerate of matter. If curvature of space with an incompleteness $I^{2}=0$ is thought of as a massless taut rope, then any ordinary curved space, given by equation (37), is as a result of a conglomerate of matter, which may be regarded as mass. Then, $I^{2} \neq 0$ provides the curvature or sagging of the rope. This interpretation indicates that

$$
m \propto \frac{1}{I^{1} I^{2}}=\frac{1}{I^{1}} \frac{\varphi}{\nabla \varphi}
$$

which indicates that mass is a form of local stretching of the rope (note that $1 / I^{2}$ is the radius of curvature of the potential field, and $1 / I^{1}=B_{\nu}$ describes the local kinematic conditions). Thus, this is a geometric extension of the space-matter field, a notion which was paradoxically first introduced by Descartes [16]. Now using Einstein's famous equation $E=m c^{2}$, and noting that $\varphi \rightarrow \pm c^{2}$ in the limiting cases described earlier, it is clear that mass can be interpreted as

$$
\begin{aligned}
m & =\frac{1}{I^{1}} \frac{1}{\nabla \varphi}=\frac{B_{v}}{\nabla \varphi}=\frac{1}{\nabla \varphi}\left\{1+\frac{1}{2}\left(\frac{\varphi}{c^{2}}\right)^{2}+\cdots\right\} \\
& =\frac{1}{\nabla \varphi}\left\{1+\frac{1}{2}\left(\frac{\nu}{c}\right)^{2}+\cdots\right\}
\end{aligned}
$$

This can become the definition for the local mass, which has the unit of $\mathrm{s}^{2} / \mathrm{m}$ : the rate of sagging of the curve, which may be expressed in kilogram. Using Einstein's equation

$$
E I^{1} \frac{\nabla \varphi}{\varphi}=1
$$

With $m$ given by equation (39), one would arrive at Einstein's equation with $\varphi \rightarrow c^{2}$. It should be noted that, in general, $\varphi=c v$, unless all mass is converted to pure energy. With net gravitational implosion $\varphi<0$, indicating that some of the energy is converted into mass. This would be very small unless $\|v\| \rightarrow c, v<0$, and in the extreme case, $\varphi \rightarrow-c^{2}$ means that all energy is converted to mass. These two extreme conditions are unattainable as described earlier. It is also clear that for energymass balance to hold

$$
\begin{aligned}
& I^{2-}=\left\|\left.I^{2}\right|_{\varphi<0}\right\|=\left\|\left.I^{2}\right|_{\varphi>0}\right\|=I^{2+} \\
& I^{2-} \times I^{2+} \neq 0: \nabla \varphi^{-} \times \nabla \varphi^{+} \neq 0 \\
& I^{1}>0 \text { for } v \rightarrow \pm c
\end{aligned}
$$


Thus, the imploding matter towards a negative potential (i.e. gravitational) should undergo fusion, and the energy released would propagate electromagnetically with a net positive potential. Thus, the extreme case of $\varphi \rightarrow-c^{2}$ cannot be reached, since the converted null space, described in section 9 necessitates $\nabla s \neq 0$. The value of $\nabla c=0$ is also unattainable, and energy will convert to mass immediately that such a space is created (meaning that the limit $\varphi \rightarrow c^{2}$ is also theoretical). Therefore, black holes should radiate electromagnetic energy in planes orthogonal to the curvature of their event horizon, as indeed observed [13].

It is more natural in the analogy of the taut massless rope put forward above to use an equality in equation (38) to define mass, which would now retain the form $m=\left(1 / I^{1}\right)(\varphi / \nabla \varphi)$, which is purely geometrical and has the conventional unit of metre, in which case $E=m$.

It is clear that Newton's second axiom can also be put in geometric form, using equations (20) and (39) as

$$
\left(I^{1}\right)^{3} F=1
$$

As in the case of relativity, force has no dimensions. One may represent it as $F=\left(1 / I^{1} \nabla \varphi\right) B_{v}^{2} \nabla \varphi=$ $\left(a / I^{1} \nabla \varphi\right)=m a$ and with the earlier definition for mass, it retains the usual unit of $N$.

Now referring back to Fig. 1 and noting that for convenience the tangent $\mathrm{AD}$ may be regarded as the slope of unity (the theoretical perfection), the curved motions occur with respect to this slope. This causes the sagging of the taut rope in the aforementioned analogy (note that the curve of motion is unlikely to be circular). BD is the subtense of the angle of deviation from the slope of unity and is the versed sine of the arc $A B$, shown as AC. The deviation $B D$ tends to $\mathrm{AC}$ in the limit, where clearly according to section $10, \nabla c \rightarrow 0$. By virtue of corollary IV in the Principia [5], these displacements are caused by the impulses acting at point $\mathrm{B}$, and it is clear that in the limit, here set as $\Delta t \rightarrow 1 / c$, they will have a continuous nature. These impulses are interpreted as the centripetal force in the Principia, being equivalent to the force $\mathrm{F}$ in equation (42). Because the incompleteness is measured from the slope of unity, where the converted space (mentioned earlier) is void of resistance to electromagnetic motion, other bodies are drawn away to undertake curvilinear paths, described by $I^{2}$. The ratio of motions of these bodies to that on the slope of unity is the versed sine of the arc $\mathrm{AB}$, as described earlier. This means that the ratio of intervals of local time to the time of the stationary system should be given by $\left(I^{1}\right)^{3}=\left\{1-\left(\varphi / c^{2}\right)^{2}\right\}^{3 / 2}$, which is precisely that in equation (42). This, in fact, is the general case of a particle/body in curvilinear orbital motion about a massive body (relative to the particle itself), subject to a rectilinear motion due to the action of significant external forces, in which the particle describes areas along the orbit in equal times (i.e. all the observed planetary motions). An important point to note is the unified nature of equation (42), irrespective of the form of potential $\varphi$. It indicates that force is a local interpretation of geometry of space as a deviation from uniformity, or in other words, as an incompleteness.

\section{CONCLUDING REMARKS}

The special theory of relativity declares the constancy of speed of light in vacuo and the Galilean principle of relativity both as laws of physics. To limit the motion of all matter within the light cone, and remain true to the principle of relativity, the unbounded Galilean transformation was replaced by the Lorentzian boost. The introduction of the special theory of relativity had profound effect in rationalization of many unnatural conclusions of Newtonian physics at limiting conditions, a number of which are referred to in section 3 . However, the use of the Lorentzian boost function, together with the constancy of the speed of light led to the retention of singular behaviour at limits of motion. Einstein's quest to extend the theory to the case of accelerated motion in non-inertial frames of reference led him to abandon his atomistic view. Through the exposition of the special theory he had unified the action of electromagnetic fields with the atomistic nature of the classical physics. He was struck by the views of Mach, and interpreted them in the form of Mach's principle, described in the previous section. This allowed him to develop a new theory for gravity, finally leading to curved spacetime and motion in non-inertial frames of reference (i.e. the general theory of relativity [17]). Einstein, as a field theorist, favoured the description of space-time in terms of action of weak gravitational fields, and accepted the limitations of the law of constancy of the speed of light. However, the retention of the Lorentzian boost function kept the concept of atomism at the heart of the special theory. This emanated from his desire to describe all forms of potential action by a unified theory for gravity. The open loop nature of such a field, and the lack of an explanation for observed dispersive action have left the special theory open to some interpretations, such as anamolous dispersion of accelerated pulses of light. For one thing, the aforementioned singular behaviour had remained. For another, the atomistic nature of the special theory somewhat decouples it 
from the general theory through retention of some (in the words of D'Alembert and interpretations of Mach) imponderable quantities such as mass. Furthermore, some opportunities may not have been realized. For example, using the Galilean principle of relativity (noting that there is no distinction between the states of absolute rest and absolute motion) instantaneity was set as the time of the stationary system, but this was not used to replace the concept of time as the interaction of light with space, which would necessitate its deviation from that in vacuo. In this manner, time would be implicit in the fabric of space, and manifested by variations in speed of light at any position, as indeed is observed as rays of light bend in travelling through different media. This undertaking necessitates the description of a closed loop spacematter field, in accord with Mach's principle, which renders this principle the status of a law of physics. The aforementioned field will then be bounded in line with the light cone and behave as a dispersive action in what is defined as a converted null space to replace the concept of vacuo at one extreme, and strongly attractive at the other extreme to accommodate very strong gravitational fields. In between, the field is an amalgam of gravitating action of matter and dispersive nature of electromagnetic potential in the form of light. The behaviour of light in such fields conforms to Huygens' principle. These additions, embodied in the principle of incompleteness result from an interpretation of the special theory and underpin the same to lead naturally to the general theory as any theory for kinematics should extend to the general case of dynamics.

\section{REFERENCES}

1 Galilei, G. Discourses Concerning Two New Sciences, 1638 (Elsevier, Leiden).

2 Lorentz, H. Versuch einer Theorie der elektrischen und optischen Erscheinungenin bewegten Korpen, 1895 (E. J. Brill, Leiden).

3 Maxwell, J. C. A Dynamical Theory of the Electromagnetic Field, 1865 (Royal Society, London).

4 Einstein, A. Zur Elektrodynamik bewegten Körper. Ann. Phys., 1905, 17, 891-921.

5 Newton, I. Philosophiae Naturalis Principia Mathematica, 1687 (Royal Society, London).

6 Einstein, A. Ist die Träegheit eines Köerpers von seinem Energieinhalt abhäengig. Ann. Phys., 1905, 18, 639-641.

7 D'Alembert, J. Le R. Traité de dynamique, 1743 (Académie de Sciences, Paris).

8 Rahnejat, H. Foreword - a tribute to Jean D'Alembert and Albert Einstein: geometrical interpretation of motion: an ironic legacy of apparently irreconcilable atomistic and continuum philosophies. In Multi-body Dynamics: Monitoring and Simulation Techniques - III, (Professional Engineering Publishing Ltd, Bury St Edmunds and London, UK).

9 Minkowski, H. Space and time. In The Principle of Relativity, 1952 (Dover Publication, New York).

10 Cauchy, A. L. Cours d'analyse, 1821 (Ecole Polytechnique, Paris).

11 Rahnejat, H. Relativity: 300 years from a principle to reality. Proc. Instn Mech. Engrs, Part K: J. Multi-body Dynamics, 2001, 215, 1-6.

12 Wang, L. J., Kuzmich, A., and Dogariu, A. Gain-assisted superluminal light propagation. Nat. Lett., 2000, 406, 277-279.

13 Revnivtsev, M. G., Churazov, E. M., Sazonov, S. Y., Sunyaev, R. A., Lutovinov, A. A., Gilfanov, M. R., Vikhlinin, A. A., Shtykovsky, P. E., and Pavlinsky, M. N. Hard X-ray view of the past activity of Sgr A* in a natural Compton mirror. Eur. J. Astron. Astrophys., 2004.

14 Huygens, C. H. R. Traité de la lumière, 1690 (Van der Aa, Leiden).

15 Mach, E. Die Mechanik in ihrer Entwicklung: histerischkritisch dagerstellt, 1883 (Brockhaus, Leipzig).

16 Descartes, R. Principia Philosophiae, 1644 (Elsevier, Amsterdam).

17 Einstein, A. Die Grundlagen der allgemeinen Relativitatstheorie. Ann. Phys., 1916, 49, 769-822.

\section{APPENDIX}

\section{Notation}

a acceleration

$B_{v} \quad$ Lorentzian boost

$c \quad$ speed of light

E energy

$F$ force

$G \quad$ universal gravitational constant

$I^{1} \quad$ first incompleteness $=\mathrm{B}_{v}^{-1}$

$I^{2} \quad$ second incompleteness

$M \quad$ source mass

$m \quad$ mass

$R \quad$ radius

$r \quad$ distance from source mass

$s \quad$ displacement or arc of motion

$t \quad$ time

$v \quad$ velocity

$\Delta t \quad$ increment of time

$\varphi \quad$ potential

$\mu \quad$ rest mass

$\tau \quad$ local time

$\xi^{i} \quad$ time coordinate, $i=0$

$\zeta=\xi^{i} \quad$ spatial coordinates, $i=1 \rightarrow 3$

$\nabla \quad$ gradient

$\nabla \bullet \quad$ divergence

$\nabla^{2} \quad$ Laplacian 